\title{
A Model For THE REFlectance of THIN LAYERS, FRONTS, AND INTERNAL WAVES AND ITS INVERSION
}

\author{
By J. Ronald V. Zaneveld \\ and W. Scott Pegau
}

\begin{abstract}
$\mathrm{T}$ HE INTERPLAY OF physical and biogeochemical processes in the ocean can result in well-defined vertical gradients and maxima in biological properties. When these gradients and maxima exist near the sea surface, it is possible to use satellite or airborne remote sensing to infer physical structure of thin layers, fronts, and internal waves within the ocean. The necessary conditions for this application of remote sensing are tied to the Inherent Optical Properties (IOP) of the water and to the local concentration (layers) of particles within the optical viewing range of remote sensing systems. In this paper, we use a two-stream radiative transfer model to demonstrate that discrete layers of particles (usually phytoplankton) can provide sufficient remotely sensed reflectance to resolve associated subsurface physical features such as the depth of specific layers of optical materials, depth and position of frontal boundaries, and the wavelength and amplitude of nearsurface internal waves. This inversion of remotely sensed optical properties to obtain information on physical structure depends on the association of in-water biological, optical, and physical structure (for specific examples, see other articles in this issue).

To understand when the conditions are right for such a visualization of physical properties via biooptical remote sensing, we need to look at the vertical structure of the optical properties in relation to the physical properties. The IOP (Preisendorfer, 1976) govern the radiative transfer in the ocean. They are not directly dependent on the external lighting conditions. These IOP are due to particulate matter, dissolved substances, and water itself. Of these three, it is the particulate matter, primarily the
\end{abstract}

J. Ronald V. Zaneveld and W. Scott Pegau, College of Oceanic and Atmospheric Sciences, Ocean. Admin. Bldg. 104, Oregon State University. Corvallis, OR 97331. USA. phytoplankton, that determine the near-surface vertical structure of the IOP. The IOP are the absorption coefficient $\mathrm{a}(\lambda, z)$, the beam attenuation coefficient $c(\lambda, z)$, and the volume scattering function $\beta(\theta, \lambda, z)$ (for definitions see Jerlov. 1976; Gordon et al., 1979), where $\lambda$ is the wavelength of light, $\mathrm{z}$ is the depth, and $\theta$ is the scattering angle. The scattering coefficient $b(\lambda, z)$ is the integral over all directions of the volume scattering function, and the attenuation coefficient is the sum of the absorption and scattering coefficients, so that in practice only the absorption coefficient and the volume scattering function need to be known to describe the behavior of radiance in a medium, if we ignore the usually minor contributions of polarization, inelastic scattering, and internal sources.

The diffuse or irradiance reflectance $\mathbf{R}$ at a depth $\mathrm{z}$ is defined as the ratio of the upwelling irradiance $E_{u}$ and the downwelling irradiance $E_{d}$. Hence $R(z)=$ $E_{u}(z) / E_{d}(z)$. This parameter has been extensively modeled (for example Gordon et al., 1988; Morel, 1988; Gordon, 1989; Morel and Gentili, 1991), primarily because of its ease of measurement since the irradiance sensor does not require absolute calibration. Remote sensing satellites sense radiance rather than irradiance, so that the models were subsequently modified to look at the ratio of the upwelling radiance $L_{u}$ and the downwelling irradiance (Zaneveld, 1982, 1995; Gordon et al., 1988; Gordon, 1992; Morel and Gentili, 1993). The ratio $R_{r}(z)=L_{u}(z) / E_{d}(z)$ as used in the later papers is often called the remote sensing reflectance. Instrumentation was also developed to measure the upwelling radiance spectrum.

In this paper we develop a simple model to study under what circumstances features like the slopes of fronts, amplitudes of internal waves, and thicknesses of thin layers can be determined from remote sensing. Forward radiative transfer models are available (e.g., Mobley et al., 1993) for the determination of reflectance in stratified optical systems. Calculating the reflectance for an arbitrary vertical optical structure is thus possible. We are interested here in developing an inversion scheme, which requires a simpler two-stream model. These simpler models calculate the fluxes in the upward and downward directions only. Full radiative transfer modeling is less well suited to this task than twostream models because the results of the full radiative transfer models cannot be mathematically inverted without first fitting empirical models to the results. Because of their simple mathematical structure, twostream models lend themselves well to the inversion task. On the other hand, results are only approximate, and careful attention must be paid to the conditions under which they can be applied.

\section{A Two-Stream Model for Physical Structure}

To model the reflectance for various optical stratifications due to physical structure, we employ a simple two-stream model such as that used by Philpot and Ackleson (1981), Philpot (1987, 1989), Maritorena et al. (1994), and others to study the effect of bottom albedo on the remotely sensed reflectance. These approaches all have common two-stream assumptions (Preisendorfer, 1976); it is assumed that there is some backscattering parameter $\mathrm{B}(\mathrm{z})$ that characterizes the redirection of light upward, and that there is some attenuation coefficient $g(z)$ that characterizes the round trip attenuation from the surface to a given depth $\mathrm{z}$ and back. The paper by Maritorena et al. (1994) provides an excellent discussion of the errors resulting from these assumptions. It should be noted that the diffuse reflectance and the remote sensing reflectance can be modeled by the same mathematical 
formalism, but the values of the parameters must be changed.

We will divide the water column into $\mathrm{N}$ homogeneous layers. The value of the IOP in each layer will be determined by the desired vertical distribution of optical properties. In accordance with the simple model we will assume that each layer has a diffuse attenuation coefficient $g(z)$ that describes the round trip attenuation of the up welling and downwelling irradiance through the layer. We will also assume that the irradiance reflects according to some backscattering coefficient $\mathrm{B}(\mathrm{z})$. With the above very simple notation, one can write:

$\mathrm{E}_{\mathrm{u}}\left(0^{-}\right)=\mathrm{E}_{\mathrm{J}}\left(0^{-}\right)$

$$
\dagger \int_{,}^{x} B(z) e^{-2 \tau}(z) d z
$$

where

$$
\tau g(z)=\int_{,}^{\prime} g(z \pi) d z \pi .
$$

$E_{u}\left(0^{-}\right)$and $E_{d}(0)$ are the upwelling and downwelling irradiances just below the surface, and the parameters $B(z)$ and $g(z)$ are apparent optical properties because they depend both on the inherent optical properties and the radiance distribution. The integrals can be broken down into sums over a number of depth intervals if the $B(z)$ and $g(z)$ parameters are assumed to be constant in each depth interval. The $n$th interval covers depths from $z_{n}$ to $z_{n+1}$. with $\Delta z_{n}=z_{n+1}-z_{n}$, and has optical properties of $B_{n}$ and $g_{n}$. Substitution into equation (1) and integration then yields:

$$
\begin{aligned}
& R\left(0^{-}\right)=\frac{E_{u}\left(0^{-}\right)}{E_{d}\left(0^{-}\right)}=\sum_{n=1}^{N} \int_{L_{n}}^{\iota_{n+1}} B_{n} \\
& \times \exp \left(-2 \int_{0}^{\iota_{n}} g\left(z^{\prime}\right) d z^{\prime}\right) \\
& \times \exp \left(-2 g_{n} \int_{\iota_{n}}^{\lambda_{n+1}} d z^{\prime}\right) \\
& =\sum_{n=1}^{N}\left\{\frac{B_{n}}{2 g_{n}}\left[1-\exp \left(-2 g_{n} \Delta z_{n}\right)\right]\right. \\
& \left.\quad \times \exp \left(-\sum_{i=1}^{n-1} 2 g_{i} \Delta z_{1}\right)\right\}
\end{aligned}
$$

We note that the term

$$
\mathrm{T}_{\mathrm{n}}^{2}=\exp \left(-\sum_{\mathrm{i}=1}^{\mathrm{n}-1} 2 \mathrm{~g}_{1} \Delta \mathrm{z}_{\mathrm{i}}\right)
$$

is the attenuation of light to the top of the nth layer and back to the surface. The reflectivity of layer $n$ if it were at the surface would be:

$$
\mathrm{R}_{\mathrm{sn}}=\frac{\mathrm{B}_{\mathrm{n}}}{2 \mathrm{~g}_{\mathrm{n}}}\left[1-\exp \left(-2 \mathrm{~g}_{\mathrm{n}} \Delta \mathrm{z}_{\mathrm{n}}\right)\right]
$$

Equation (3) can then be simply rewritten as:

$$
R\left(0^{-}\right)=\sum_{n=1}^{N}\left(T_{n}^{2} R_{s n}\right)
$$

If the layer in equation (5) were infinitely thick, the reflectance would be:

$$
R_{x_{n}}=\frac{B_{n}}{2 g_{n}}
$$

Equation 7 is important because it allows us to measure or calculate the ratio of the parameters $B_{n}$ and $g_{n}$ for given IOP and surface radiance distribution.

With this notation we can rewrite equation (6) as:

$$
R\left(0^{-}\right)=\sum_{n=1}^{N} R_{x_{n}}\left(T_{n}^{2}-T_{n+1}^{2}\right) .
$$

The formulations in equations (6) and (7) can also include the bottom. In those cases the reflectance for the deepest layer, $R_{\infty N}$, is simply the bottom albedo.

Combining equations (7) and (3) for two layers, with the second layer being optically infinitely deep, we obtain:

$$
\begin{aligned}
R\left(0^{-}\right)=R_{x_{1}}[ & \left.-\exp \left(-2 g_{1} \Delta z_{1}\right)\right] \\
& +R_{\times 2} \exp \left(-2 g_{1} \Delta z_{1}\right) .
\end{aligned}
$$

Depending on the thickness of the first layer, $\Delta z_{1}$, the reflectance can vary from $R_{\infty / 1}$ to $R_{\infty 2}$. If we now look at the $\mathrm{N}$ layered model, but only vary the thickness of the first layer, we can deduce from equation (3) that:

$$
\begin{aligned}
R\left(0^{-}\right)= & R_{x_{1}}\left[1-\exp \left(-2 g_{1} \Delta z_{1}\right)\right] \\
+ & \exp \left(-2 g_{1} \Delta z_{1}\right) \\
\times \sum_{n=2}^{N} & \left\{\frac{B_{n}}{2 g_{n}}\left[1-\exp \left(-2 g_{n} \Delta z_{n}\right)\right]\right. \\
& \left.\quad \times \exp \left(-\sum_{i=2}^{n-1} 2 g_{1} \Delta z_{i}\right)\right\} .
\end{aligned}
$$

By setting

$$
\begin{aligned}
\mathrm{R}_{2 N}=\sum_{\mathrm{n}=2}^{\mathrm{N}} & \left\{\frac{\mathrm{B}_{\mathrm{n}}}{2 \mathrm{~g}_{\mathrm{n}}}\left[1-\exp \left(-2 \mathrm{~g}_{\mathrm{n}} \Delta z_{\mathrm{n}}\right)\right]\right. \\
& \left.\times \exp \left(-\sum_{\mathrm{i}=2}^{\mathrm{n}-1} 2 \mathrm{~g}_{\mathrm{i}} \Delta z_{\mathrm{i}}\right)\right\},
\end{aligned}
$$

We then get that:

$$
\begin{aligned}
\mathrm{R}\left(0^{-}\right)=\mathrm{R}_{x_{1}} & {\left[1-\exp \left(-2 \mathrm{~g}_{1} \Delta \mathrm{z}_{1}\right)\right] } \\
+ & \mathrm{R}_{2 \mathrm{~N}} \exp \left(-2 \mathrm{~g}_{1} \Delta \mathrm{z}_{1}\right)
\end{aligned}
$$

so that by comparison with equation (9) it is seen that the entire structure of layers 2 through $\mathrm{N}$ can be considered to be a single layer (layer $2 \mathrm{~N}$ ) as far as the dependence of the irradiance reflectance on a changing thickness of the first layer is concerned. Layer $2 \mathrm{~N}$ is infinitely deep. Solving for the optical depth of the first layer gives:

$\mathrm{g}_{1} \Delta \mathrm{z}_{1}=-0.5 \ln \left[\frac{\mathrm{R}\left(0^{-}\right)-\mathrm{R}_{\times 1}}{\mathrm{R}_{2 \mathrm{~N}}-\mathrm{R}_{\times 1}}\right]$

Equation (13) shows that if we can measure $R_{\infty 1}$ and $R_{2 N}$, we can determine the variable optical depth of the first layer, $\Delta z_{1} g_{1}$, if $R(0)$ is measured as a function of location. This concept is critical to what follows. If we have a stratified ocean in which a layer of varying thickness with homogeneous properties overlies the remainder of the ocean, which can contain any number of layers, we can then determine the optical thickness of the first layer, g, $\Delta z_{1}$. provided the optical properties of the first layer do not covary with the optical properties of the layers below it. To do so, we must be able to measure the reflectance of the first layer where it is optically infinitely deep $\left(R_{\infty 1}\right)$. and the reflectance of the combination of the second through Nth layer in a location where the first layer does not exist $\left(R_{2 N}\right)$. With the above equations in hand, a number of geometries can be resolved.

\section{Reflectance of Physical Features}

\section{Thin Layers}

Generation mechanisms of the thin layers are discussed elsewhere in this volume. The high concentration of biological materials in these layers results in increases in the scattering and $a b$ sorption characteristics in these layers. Thin layers are usually present offshore 
of coastal upwelling fronts and at the bottom of the mixed layer. This was observed by Zaneveld and Pak (1979), who discussed "optical amplification" of physical features. At the time, only beam attenuation could be measured in a continuous vertical profile. Recent advances in instrumentation (Moore et al., 1992; Zaneveld et al., 1994) now allow us to determine the spectral absorption, attenuation (and hence scattering) coefficients in a continuous vertical profile at the same time, and space scales as the physical parameters. By means of filtering the intake of the flow-through in situ instrumentation, it is even possible to separate the effects due to dissolved and particulate components. It is, of course, this instrumental advance that has sparked the current interest in the interaction of the biology, physics, and optics of thin layers.

We can model a thin layer as one in which the inherent optical properties are much larger than in the water immediately above and beneath it. Equation (3) would thus apply. From equations (3) and (12) we see that the influence of the thin layer on the reflectance depends exponentially on its depth and on the reflectance of the thin layer if it were infinitely thick and at the surface (as expressed in Eq. 7). From equation (13) we see that we can only invert for the depth of the thin layer if $R_{2 N}$ is known, or we can solve for the optical properties via $R_{2 N}$ if the depth is known.

\section{Fronts}

At oceanic fronts the physical structure is usually accompanied by strong gradients in optical properties (Zaneveld and Pak, 1979). The front is modeled as a wedge of homogeneous watermass 1 overlying stratified watermass 2 . Figure 1 shows the structure of the hypothetical front. Referring to equation (12), watermass 1 would have a reflectance of $R_{\infty 1}$, if it were infinitely deep, and watermass 2 would have a reflectance of $R_{2 N}$. Note that watermass 2 does not need to be vertically homogeneous. If locations are known where the pure watermasses occur, $R_{\infty 1}$ and $R_{2 N}$ can be measured at those locations. Such a location is found for watermass 1 at a distance from the front where the upper layer is thick enough to have become optically infinitely deep. It can be

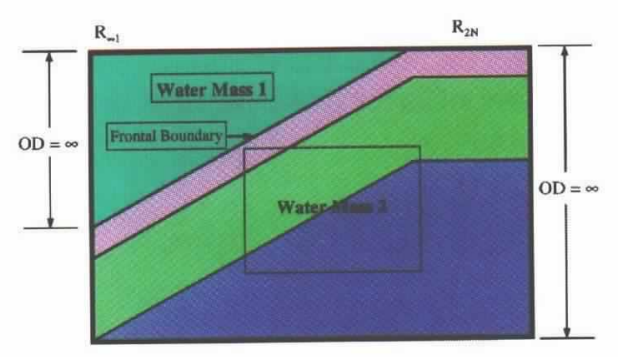

Fig. 1: Structure of a hypothetical front. Watermass 1 is physically homogeneous. At some distance from the front, the optical depth (OD) of watermass 1 becomes infinitely large. The reflectance $R_{\infty 1}$ is measured here. The reflectance of the stratified watermass 2 is measured at the other side of the front. For further details, see the text.

shown that when watermass 1 overrides stratified watermass 2 and forces it to subside, the layers are stretched so that they become thinner perpendicular to the boundary of the two watermasses, but retain the same thickness in the vertical direction. The $R_{2 N}$ that is valid when the layers 2 through $\mathrm{N}$ has horizontal boundaries is thus also valid for inclined boundaries.

The vertical structure of a front can then be determined from optical remote sensing using the following approach. The reflectance is measured on either side of the front at a location where the reflectance has become constant as a function of distance from the front. This determines parameters $R_{\infty 1}$ and $R_{2 N}$ in equation (13). The reflectance is then measured as a function of location across the front. Equation (13) is then applied to determine the optical depth at each location. The actual depth can then be determined if $g_{1}$ is known. Maritorena et al. (1994) have shown that $\mathrm{g}_{1}$ is 1.01 to 1.33 times greater than the downward diffuse attenuation coefficient $K_{d}$ (which in turn is a few percent different from the total diffuse attenuation coefficient). Fairly good algorithms for the determination of $\mathrm{K}(490)$ from remote sensing exist (Austin, 1981), thus at the "pure" watermass 1 location the K(490) of the overriding watermass 1 can be determined. With the use of relations between $\mathrm{g}$ and $\mathrm{K}$ as in Maritorena $e t$ al. (1994), it is then possible to determine the depth of the interface using equation (13).

\section{Sloping Bottoms}

It is interesting to note that the same procedure can be applied to a watermass of varying depth overlying a bottom. In that case $R_{2 N}$ is the bottom albedo, and equation (13) can be used to derive bottom depth by the same approach. This method was used by Philpot and Ackleson (1981) to experimentally determine bottom depth using a known bottom albedo. A similar approach was used by Maritorena et al. (1994). It does not appear to have been recognized that $R_{2 N}$ and hence the albedo can be determined remotely in very shallow water. If the nature of the bottom and therefore the albedo does not change, the bathymetry can be determined entirely by passive remote sensing using an approach very similar to that for fronts, without any a priori knowledge of the bottom albedo. In the bottom case we should measure the reflectance in very shallow water close to shore. This will be reflectance $R_{2 N}$. The layer $2 \mathrm{~N}$ thus consists of a thin layer of water and the bottom. Physically homogeneous watermass 1 overlies this layer, and its thickness constitutes the bathymetry. The thickness of watermass 1 and the bathymetry are then determined in an identical way to that described above. The key here is that no a priori knowledge of the bottom albedo is necessary. The only requirement is that for accurate bathymetry the albedo must not change. This method could be of use in inaccessible areas. Tidal range can also be determined in this way.

\section{Internal Waves}

It is quite common for internal waves to occur at density interfaces. In that case internal waves will continuously change the depth of a layer, which will modify the reflectance. Such an event was observed at East Sound where the ocean color was seen to change from light whitish green to dark green with a periodicity of minutes. Unfortunately, no time series record was obtained. With the use of equation (12), the dependence of the irradiance reflectance on an internal wave can readily be modeled. Substituting

$$
\Delta \mathrm{z}_{1}(\mathrm{t}, \mathrm{x})=\mathrm{z}_{2}+\mathrm{A} \cos (\omega \mathrm{t}+\mathrm{kx}),
$$

where $\mathrm{A}$ is the amplitude, $\omega$ the frequency, and $\mathrm{k}$ the wavenumber of the in- 
ternal wave, into equation (12), leads to the desired relationship:

$$
\begin{aligned}
& \mathrm{R}\left(0^{-}, \mathrm{t}, \mathrm{x}\right)=\mathrm{R}_{\times 1} \\
& \times\left(1-\exp \left\{-2 \mathrm{~g}_{1}\left[\mathrm{z}_{2}\right.\right.\right. \\
&+\mathrm{A} \cos (\omega \mathrm{t}+\mathrm{kx})]\}) \\
&+\mathrm{R}_{2 \mathrm{~N}} \exp \left\{-2 \mathrm{~g}_{1}\left[\mathrm{z}_{2}\right.\right. \\
&\quad+\mathrm{A} \cos (\omega \mathrm{t}+\mathrm{kx})]\} .
\end{aligned}
$$

Because the amplitude appears in the exponent. there can be a considerable nonlinear influence on the reflectance at the surface.

Inversion of the internal wave case is interesting because in this case the layer $2 \mathrm{~N}$ does not occur at the surface, in contrast to the frontal case. The amplitude of the internal wave can still be solved for, however, because the reflectance $R_{2 N}$ does not need to be known, as will be demonstrated. We take layer $2 \mathrm{~N}$ to consist of the water mass structure from the top of the interface on which the wave rides, downward to infinity. At location, $\mathrm{x}_{1}$, where the wave is closest to the surface, the depth of the density and optical interface on which the internal wave rides is $z_{2}-A$, and the reflectance is $R\left(x_{1}\right)$. At location $x_{2}$, where the wave is furthest from the surface, the depth of the internal wave is $z_{2}+A$, and the reflectance is $R\left(x_{2}\right)$. Applying equation (13) at $x_{1}$ and $x_{2}$ and subtracting then gives:

$$
\begin{aligned}
& {\left[\left(\mathrm{z}_{2}+\mathrm{A}\right)-\left(\mathrm{z}_{2}-\mathrm{A}\right)\right] \mathrm{g}_{1}} \\
& \quad=-0.5 \ln \left[\frac{\mathrm{R}\left(\mathrm{x}_{2}\right)-\mathrm{R}_{\times 1}}{\mathrm{R}_{2 \mathrm{~N}}-\mathrm{R}_{\times 1}}\right] \\
& \quad+0.5 \ln \left[\frac{\mathrm{R}\left(\mathrm{x}_{1}\right)-\mathrm{R}_{\times 1}}{\mathrm{R}_{2 \mathrm{~N}}-\mathrm{R}_{\times 1}}\right],
\end{aligned}
$$

or

$$
2 \mathrm{Ag}_{1}=-0.5 \ln \left[\frac{\mathrm{R}\left(\mathrm{x}_{2}\right)-\mathrm{R}_{r-1}}{\mathrm{R}\left(\mathrm{x}_{1}\right)-\mathrm{R}_{r \cdot 1}}\right] \text {. }
$$

The optical amplitude of the internal wave can thus be determined, and the actual amplitude can be approximated if $\mathrm{g}_{1}$ can be determined via the diffuse attenuation coefficient $\mathrm{K}$.

\section{Discussion}

The applicability of the inversion is by no means universal and is dependent on several factors. The difference in the reflectances of the watermasses, as in equation (13), must be large enough to obtain meaningful results. Different remote sensing instruments have vastly different sensitivities in terms of determining the reflectances. The error in determining the reflectances greatly influences the ability to invert. Finally, the parameter $g_{1}$ must be determined to obtain the actual depth. An excellent discussion on the dependence of this parameter on external lighting conditions and the IOP can be found in Maritorena $e t$ al. (1994). As stated above, they concluded that $\mathrm{K}<\mathrm{g}_{1}<1.32 \mathrm{~K}$. This parameter alone can thus lead to a $15 \%$ error if we assume that $\mathrm{g}_{1}=1.16 \mathrm{~K}$.

Further work is needed to test the approach outlined above using satellite or aircraft optical remote sensing. At present, satellites typically have pixel sizes on the order of $1 \mathrm{~km}$, so that they could only be used for the largest scale features. Aircraft remote sensing would thus be more appropriate for the optical detection of physical features. It would be useful to determine the optical and physical structure of the ocean during remote sensing in order to assess the viability of the approach.

\section{Acknowledgement}

This work was supported by the Environmental Optics branch of the Office of Naval Research and the Ocean Biology/Biogeochemistry program of the National Aeronautics and Space Administration.

\section{References}

Austin. R.W., 1981: Remote sensing of the diffuse attenuation coefficient of ocean water. In: Proceedings of the 29th Symposium of the AGARD Electromagnetic Wave Propagation Panel. 18-1 to 18-9.

Gordon. H.R., R.C. Smith and J.R.V. Zaneveld, 1979: Introduction to ocean optics. In: Ocean Optics VI, Proc. SPIE 208, 14-55.

1989: Dependence of the diffuse reflectance of natural waters on the sun angle. Limnol. Oceanogr. 34, 1484-1489.
O.B. Brown. R.H. Evans, J.W. Brown. R.C. Smith, K.S. Baker and D.K. Clark, 1988: A semi-analytic radiance model of ocean color. J. Geophys. Res.. 9.3D, $10,909-10.924$.

1992: Diffuse reflectance of the ocean: influence of nonuniform pigment profile. Appl. Opt.. 31, 2116-2129.

Jerlov. N.G., 1976: Marine Optics. Elsevier Oceanography Series, vol. 14. Elsevier, Amsterdam, $231 \mathrm{pp}$.

Maritorena, S., A. Morel and B. Gentili, 1994: Diffuse reflectance of oceanic shallow waters: influence of water depth and albedo. Limnol. Oceanogr., 39, 1689-1703.

Mobley, C.D., B. Gentili, H.R. Gordon, Z. Jin, G.W. Kattawar. A. Morel. P. Reinersman. K. Stamnes and R.H. Stavn, 1993: Comparison of numerical models for computing underwater lightfields. Appl. Opt., 3. 7484-7504.

Moore, C., J.R.V. Zaneveld and J.C. Kitchen, 1992: Preliminary results from an in situ spectral absorption meter. In: Ocean Optics XI. G.D. Gilbert. ed. Proc. SPIE 1750. 330-337.

Morel. A., 1988: Optical modeling of upper ocean in relation to its biogenous matter content (case I waters). J. Geophys. Res., 93, $749-768$.

and B. Gentili, 1991: Diffuse reflectance of oceanic waters: its dependence on sun angle as influenced by the molecular scattering contribution. Appl. Opt., 30, 4427-4438. and B. Gentili. 1993: Diffuse reflectance of oceanic waters. II. Bidirectional aspects. Appl. Opt., 32, 6864-6879.

Philpot, W.D.. 1987: Radiative transfer in stratified waters: a single-scattering approximation for irradiance. Appl. Opt., 26. 4123-4132.

1989: Bathymetric mapping with passive multispectral imagery. Appl. Opt., 28, 1569-1578.

and S. Ackleson, 1981: Remote sensing of optically shallow, vertically inhomogeneous waters: a mathematical model. NASA Conference Publication 2188, Proceedings from Chesapeake Bay Plume Study Superflux 1980, Williamsburg. VA.

Preisendorfer, R.W., 1976: Hydrologic Optics (in 6 volumes). Dept. of Commerce, NOAA

Zaneveld, J.R.V., 1982: Remotely sensed reflectance and its dependence on vertical structure: a theoretical derivation. Appl. Opt., 2I, 4146-4150.

1995: A theoretical derivation of the dependence of the remotely sensed reflectance on the inherent optical properties. J. Geophys. Res., 100, 13,135-13,142.

J.C. Kitchen and C.C. Moore. 1994: Scattering error correction of reflecting tube $a b-$ sorption meters. In: Ocean Optics XII. S. Ackleson. ed. Proc. SPIE vol. 2258, 44-55. and H. Pak, 1979: Optical and particulate properties at oceanic fronts. J. Geophys. Res., 4.7781-7790. $\square$ 